Cite this: Phys. Chem. Chem. Phys., 2011, 13, 17130-17134

\title{
Stabilization of chiral molecules by decoherence and environment interactions in the gas phase
}

\author{
Isabel Gonzalo $* a^{a}$ and Pedro Bargueño ${ }^{b}$ \\ Received 27th May 2011, Accepted 1st August 2011 \\ DOI: $10.1039 / \mathrm{c} 1 \mathrm{cp21729a}$
}

We study the tunnel dynamics of a chiral molecule between its left $(L)$ and right $(R)$ conformations, under the global effect of collisional decoherence together with the effect of a mean-field generated by the environment where an energetic difference, $K$, between homochiral and heterochiral interactions is assumed. We show that this decoherence leads unavoidably to equal populations of the $L$ and $R$ chiral conformations even for a high enough value of $K$ which tends to keep localized an initial chiral state. However, we also show that $K$ contributes to the stabilization of an initial $L$ or $R$ state for times that could be many orders of magnitude larger than the tunneling time, in the case the decoherence rate is much greater than the tunneling rate. In this case, an estimation of this stabilization time and a critical tunneling time is made. Even in the case in which the tunneling rate is greater than the decoherence rate, the effect of $K$ is to keep localized the initial chiral state for times greater than the tunneling time. A possible slight chiral asymmetry is also considered.

\section{Introduction}

The interaction of a chiral molecule with its environment is considered the origin of the loss of coherence of the well defined parity state (superposition of chiral enantiomer states) and also the origin of the stabilization of the localized chiral states. It was Hund ${ }^{1}$ who first studied chiral stability versus tunneling in a double well potential. In the framework of the Hund model, it was found that, if the collision frequency in a gas of chiral molecules is high enough compared with the tunneling frequency between its chiral left $(L)$ and right $(R)$ conformations, tunneling coherence is quickly lost while the evolution to reach equal populations of $|L\rangle$ and $|R\rangle$ states of an initial chiral sample can become noticeably slow. ${ }^{2-6}$ Recently, Trost and Hornberger ${ }^{7}$ have identified, using molecular scattering theory, the dominant collisional decoherence mechanism which serves to explain the stabilization of chiral molecules due to a background gas.

The role of molecular interactions in the localization and stabilization of molecular chiral states versus superposition states has also been studied by several authors. ${ }^{8-12}$ Of particular interest is the work of Jona-Lasinio et al. ${ }^{13}$ in which dipole-dipole interactions in pyramidal molecules are considered by means of a mean-field approximation which gives place to a nonlinear eigenvalue equation. This approach explains the localization of the states in pyramidal

\footnotetext{
${ }^{a}$ Departamento de Óptica, Universidad Complutense de Madrid, 28040 Madrid, Spain.E-mail: igonzalo@fis.ucm.es

${ }^{b}$ Departamento de Física de Materiales, Universidad Complutense de Madrid, 28040 Madrid, Spain
}

conformations versus delocalized (superposition) ones, and also reproduces spectroscopic experimental results. However, this procedure is not always applicable to all chiral molecules where the dipole moment could not change in sign under the inversion by tunneling. Vardi ${ }^{14}$ considered in a simple and ingenious manner a mean-field approximation to account for molecular interactions in a sample of chiral molecules, obtaining a pair of nonlinear coupled equations, the nonlinearity due to the difference between homochiral and heterochiral interactions. When this difference is large enough, the population becomes self-trapped in one of the chiral states, $|L\rangle$ or $|R\rangle$. We note that in these two last approaches, the "dephasing" induced by collisions is not considered.

Finally, let us mention the attempt to explain chiral stability by means of parity violating weak interactions. These interactions would induce a slight asymmetry in the double well, favoring the stabilization of one of the enantiomers with respect to the other one. In this case and for a large enough tunneling time, the chiral states $|L\rangle$ and $|R\rangle$ become in fact eigenstates of the Hamiltonian. However, the predicted parity violating energy difference between the two enantiomers of a chiral molecule, which is of the order of $10^{-13}-10^{-20} \mathrm{eV},{ }^{15}$ has not yet been experimentally proven in spite of the numerous proposals to detect it (see for example ref. 15-22 and references therein). We must also note that its extremely tiny value seems to be not so decisive in the stabilization of localized chiral states in molecules which are not in the ultracold regime.

In this work we deal with a gas of chiral molecules where we consider for each molecule: (i) an energetic difference, $K$, between homochiral and heterochiral interactions in a 
mean-field approximation following the work of Vardi, ${ }^{14}$ and (ii) decoherence in the superposition state, due to the "dephasing" induced by collisions. We study the global effect of all these contributions as well as the relative effects of each one of them, using a two-state model where $|L\rangle$ and $|R\rangle$ states can be connected only by tunneling. We shall also consider the effect of adding a slight asymmetry in the double well due to a possible chiral influence. The model used and the assumptions made (see next section) are valid for the case of a gas and, in principle, cannot be applied to the condensed phase. We found that, for an initial chiral state $(|L\rangle$ or $|R\rangle)$, decoherence induced by collisions leads unavoidably to a racemic mixture of equal populations for $L$ and $R$ enantiomers, although at very long times in some cases. In a certain aspect, this is a different result from that found by Vardi ${ }^{14}$ and Jona-Lasinio et al., ${ }^{13}$ since, as was previously said, they do not consider such a decoherence phenomenon. However, it does not contradict the result of Jona-Lasino et al. concerning the induction of pyramidal localization states in $\mathrm{NH}_{3}$ and $\mathrm{ND}_{3}$ molecules on increasing the gas pressure. We analyze both cases, when the collisional decoherence rate is much greater than tunneling rate, and when is lower than or is of the order of tunneling. In both cases the effect of a high enough $K$ is to keep localized an initial chiral state for times greater than the tunneling time.

\section{The model and results}

In the framework of the double well model, we assume a two-state model for a chiral molecule with two possible chiral states, $|L\rangle$ and $|R\rangle$. If there are no asymmetries in the double well and the molecule is isolated, these chiral states are isoenergetic with energy $E_{0}$. These states are connected to each other by tunneling through the double well barrier, so that if $H$ is the Hamiltonian of the molecule, and following a common criterion for signs, we have $\langle L|H| R\rangle=-\delta$, with $\delta>0$. The energetic splitting between the odd and even eigenstates (superpositions of $|L\rangle$ and $|R\rangle$ states) is then $2 \delta$. A molecular state can be expressed as

$$
|\psi(t)\rangle=a_{\mathrm{L}}(t)|L\rangle+a_{\mathrm{R}}(t)|R\rangle .
$$

Let us consider the intermolecular interactions proposed by Vardi, ${ }^{14}$ introduced by means of a Hartree-Fock technique considering each molecule subjected to a mean-field resulting from all the other molecules. This field has homochiral and heterochiral contributions. For a molecule, the total Hamiltonian in the basis $\{|L\rangle,|R\rangle\}$ reads

$$
H=\left(\begin{array}{cc}
H_{\mathrm{LL}} & -\delta \\
-\delta & H_{\mathrm{RR}}
\end{array}\right)
$$

with

$$
\begin{aligned}
& H_{\mathrm{LL}}=E_{0}+\varepsilon+U_{\text {hom }}\left|a_{\mathrm{L}}\right|^{2}+U_{\text {het }}\left|a_{\mathrm{R}}\right|^{2} \\
& H_{\mathrm{RR}}=E_{0}-\varepsilon+U_{\text {hom }}\left|a_{\mathrm{R}}\right|^{2}+U_{\text {het }}\left|a_{\mathrm{L}}\right|^{2}
\end{aligned}
$$

where $U_{\text {hom }}$ and $U_{\text {het }}$ are interaction strengths due to the homochiral $(L-L, R-R)$ and heterochiral $(L-R)$ interactions with the surrounding molecules, respectively. ${ }^{14}$ We have also included a small energy, $\varepsilon$, due to a possible chiral influence.

We use the density matrix formalism to study the temporal evolution of the molecular state submitted to the above
Hamiltonian $H$ and to collisional effects of the environment molecules. Since we are interested only in the dynamics of our two-state molecular system, we use the master equation for the reduced density matrix of our molecular system. This equation is the result of tracing out the bath degrees of freedom from the equation of motion for the full density matrix of the whole system, molecule and bath. A weak enough coupling molecule-environment (as in a gas) and Markov approximation are assumed. The equation to solve is then

$$
\frac{\mathrm{d} \rho_{i j}}{\mathrm{~d} t}=-\frac{i}{\hbar}[H, \rho]_{i j}-\gamma_{i j} \rho_{i j},
$$

with $i, j=L, R$, and decay rates $\gamma_{i j}$ introduced phenomenologically.

We assume that the temperature of the sample is small enough so that the kinetic energy transferred in the collisions is sufficiently small compared to the barrier separating the chiral states $|L\rangle$ and $|R\rangle$, which can be then connected to each other only by tunneling. This assumption occurs in most cases of interest, even at ambiance temperature. We also assume thermal equilibrium in the sense that there is no net change of population levels due to collisions. In addition we consider the energies $\delta, \varepsilon$ and the difference $K \equiv U_{\text {het }}-U_{\text {hom }}$ smaller enough than the rotational levels. We then assume a two-state approach where $\rho_{\mathrm{LL}}+\rho_{\mathrm{RR}}=1$.

Denoting by $D \equiv \rho_{\mathrm{LL}}-\rho_{\mathrm{RR}}$ the population difference between $|L\rangle$ and $|R\rangle$ states, we obtain from the master eqn (5),

$$
\begin{gathered}
\frac{\mathrm{d} \rho_{\mathrm{LR}}}{\mathrm{d} t}=-\frac{i}{\hbar}\left(\delta D-2 \varepsilon \rho_{\mathrm{LR}}-K D \rho_{\mathrm{LR}}\right)-\gamma \rho_{\mathrm{LR}} \\
\frac{\mathrm{d} D}{\mathrm{~d} t}=-\frac{i}{\hbar} 2 \delta\left(\rho_{\mathrm{LR}}-\rho_{\mathrm{LR}}^{*}\right),
\end{gathered}
$$

where $\gamma$ is introduced phenomenologically to account for the decay of coherence due to the "dephasing" induced by collisions (the most frequent ones are elastic collisions). This decay is usually expressed as $\gamma=1 / T_{2}$ where the so-called "transversal" time, $T_{2}$, is here of the order of the average time between collisions. Since the eventual $\varepsilon$ is assumed to be smaller enough than the thermal energy $k T$, we can neglect the thermal equilibrium population difference, $D_{\text {eq }}$, to which $D$ relaxes in eqn (7). We note the nonlinear term dependent on $K$ in eqn (6). If this term is zero $(K=0)$, the above equations reduce essentially to those studied by several authors, ${ }^{2-7}$ some of them performed a detailed treatment of collisions. ${ }^{3,6,7}$ For $\gamma=0$ and $\varepsilon=0$ the solution of the equations was studied by Vardi, ${ }^{14}$ for different values of $K / \delta$ and different initial conditions of $|\psi(t)\rangle$. He showed that, when the nonlinearity is sufficiently large, the population is trapped in one of the wells of the symmetric double well potential. However, in our case it is easy to see that the stationary solution of eqn (6) is

$$
\rho_{\mathrm{LR}}^{\mathrm{st}}=\frac{\delta D}{K D+2 \varepsilon+i \hbar \gamma}
$$

which together with the stationary condition of eqn (7), ( $\rho_{\mathrm{LR}}^{\mathrm{st}}$ real), leads from eqn (8) to the stationary solution $\rho_{\mathrm{LR}}^{\mathrm{st}}=0$ and then to $D^{\text {st }}=0$. Thus, in the stationary state, the loss of coherence impedes the existence of superposition states, $|L\rangle$ and $|R\rangle$ states, and a mixed state is then obtained. Since the populations for $|L\rangle$ and $|R\rangle$ states become equal, 
we get an ensemble of molecules where $50 \%$ of them are the $L$ enantiomer and $50 \%$ are the $R$ enantiomer, i.e., we have a racemic mixture. This fact was called "racemization by dephasing" by Silbey and Harris. ${ }^{6}$ As we see, the decoherence due to $\gamma$ leads unavoidably to racemization whatever the value of $K$, in contrast to the result of Vardi in which such a decoherence phenomenon was not considered. For an initial pure chiral sample, the random collisions induce dephasing not only in the tunneling dynamics of each molecule interrupting its coherent tunneling, but also induce dephasing between the tunnel dynamics of different molecules. This last mechanism tends to equal the populations of $|L\rangle$ and $|R\rangle$ states, despite the tendency of the population to be trapped in one of the wells by the mean-field effect governed by $K$.

We study the solutions of eqn (6) and (7) in two different cases: $\gamma \gg \delta / \hbar$ and $\gamma \lesssim \delta / \hbar$.

- Case $\gamma \gg \delta / \hbar$. Let us first consider the usual case in which the average time between collisions is much smaller than the tunneling time, $t_{\delta}=\hbar / \delta$, of the isolated molecule, i.e., the decoherence process governed by $\gamma$ is much faster than tunneling. In this case we can consider that eqn (6) evolves much faster than eqn (7), the population difference $D$ being scarcely changed while the coherence $\rho_{\mathrm{LR}}$ reaches the stationary state given by eqn (8). Hence, by inserting eqn (8) in (7) and taking into account that the initial condition of $D(t)$ is $D_{0}$, we easily obtain

$$
t=\frac{K^{2}}{8 \delta^{2} \gamma}\left(D_{0}^{2}-D^{2}\right)+\frac{K \varepsilon}{\delta^{2} \gamma}\left(D_{0}-D\right)+\left(\frac{\varepsilon^{2}}{\delta^{2} \gamma}+\frac{\gamma \hbar^{2}}{4 \delta^{2}}\right) \ln \frac{D_{0}}{D} .
$$

We define the time $t_{\mathrm{r}}$ as the time necessary for the population difference $D$ diminishing from the initial value $D_{0}$ to the value $D_{0} / e$. It characterizes the stabilization time of an initial chiral state and is of the order of the racemization time since the coherence is completely lost when $D=0$, as shown in eqn (8). This time can be appreciated in the decay curves $D(t)$ of Fig. 1 and 2, commented below. The time $t_{\mathrm{r}}$ can be obtained from the preceding equation, and reads

$$
t_{\mathrm{r}}=\left(1-\frac{1}{e^{2}}\right) \frac{K^{2} D_{0}^{2}}{8 \delta^{2} \gamma}+\left(1-\frac{1}{e}\right) \frac{K D_{0} \varepsilon}{\delta^{2} \gamma}+\frac{\varepsilon^{2}}{\delta^{2} \gamma}+\frac{\gamma t_{\delta}^{2}}{4},
$$

where the contributions of the different physical phenomena are displayed. We note that the terms dependent on $K$ or $\varepsilon$ decrease

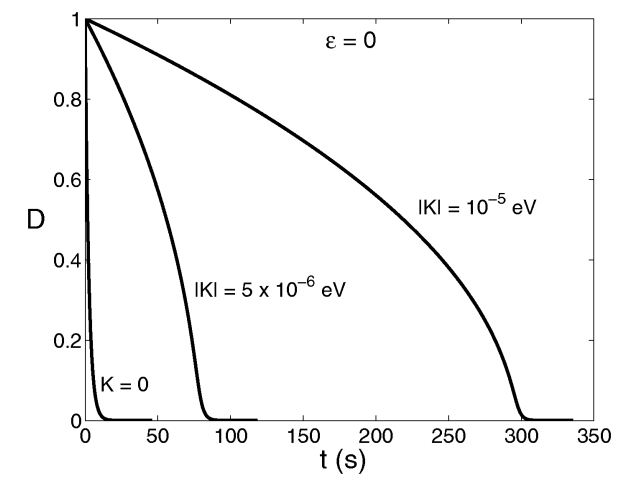

Fig. 1 Population difference $D(t) \equiv \rho_{\mathrm{LL}}(t)-\rho_{\mathrm{RR}}(t)$ as a function of time, for $t_{\delta}=10^{-4} \mathrm{~s}, \gamma=10^{9} \mathrm{~s}^{-1}$, in the case $\varepsilon=0$, and values: $K=0$, $|K|=5 \times 10^{-6} \mathrm{eV},|K|=10^{-5} \mathrm{eV}$.

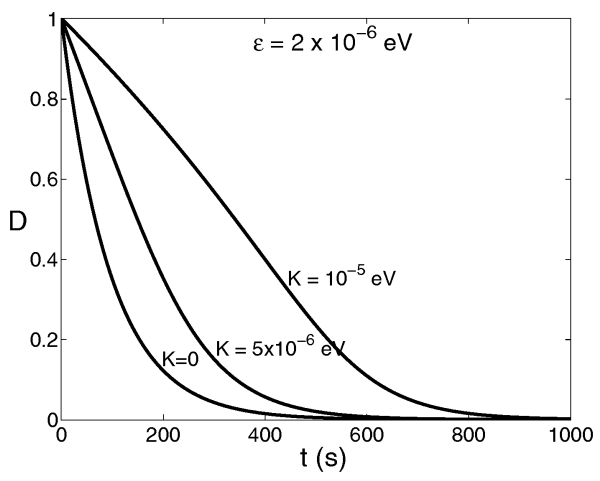

Fig. 2 Population difference $D(t) \equiv \rho_{\mathrm{LL}}(t)-\rho_{\mathrm{RR}}(t)$ as a function of time, for $t_{\delta}=10^{-4} \mathrm{~s}, \gamma=10^{9} \mathrm{~s}^{-1}$, in the case $\varepsilon=2 \times 10^{-6} \mathrm{eV}$, and values: $K=0, K=5 \times 10^{-6} \mathrm{eV}, K=10^{-5} \mathrm{eV}$.

when $\gamma$ increases, while the opposite behaviour occurs in the known last term. ${ }^{7}$ The terms proportional to $K^{2}$ or $\varepsilon^{2}$ contribute to augment the time $t_{\mathrm{r}}$ whatever the sign of $K$ or $\varepsilon$. All the terms are inversely proportional to $\delta^{2}$, i.e., we have a parabolic behavior, $t_{\mathrm{r}} \propto t_{\delta}^{2}$, which allows us to easily define a critical tunneling time $t_{\mathrm{c}}$ at which $t_{\mathrm{r}}=t_{\delta}$. It is given by

$$
t_{\mathrm{c}}=\left[\left(1-\frac{1}{e^{2}}\right) \frac{K^{2} D_{0}^{2}}{8 \hbar^{2} \gamma}+\left(1-\frac{1}{e}\right) \frac{K D_{0} \varepsilon}{\hbar^{2} \gamma}+\frac{\varepsilon^{2}}{\hbar^{2} \gamma}+\frac{\gamma}{4}\right]^{-1} .
$$

Then, for $t_{\delta}<t_{\mathrm{c}}$, we obtain $t_{\mathrm{r}}<t_{\delta}$, while for $t_{\delta}>t_{\mathrm{c}}$, we obtain $t_{\mathrm{r}}>t_{\delta}$.

In order to better analyze and estimate $t_{\mathrm{r}}$, let us consider the case in which $\varepsilon=0$. In this case $t_{\mathrm{r}}$ is reduced to

$$
t_{\mathrm{r}}=t_{K}+t_{\gamma},
$$

where we define $t_{K} \equiv\left(1-\frac{1}{e^{2}}\right) \frac{K^{2} D_{0}^{2}}{8 \delta^{2} \gamma}$ and $t_{\gamma} \equiv \frac{\gamma t_{\delta}^{2}}{4}$ from eqn (10). We must note that under the condition $\gamma \gg t_{\delta}^{-1}$ we are assuming $t_{\gamma}$ is always greater than $t_{\delta}$, as can be easily seen. We can define a critical tunneling time, $t_{K \mathrm{c}}$, at which $t_{K}=t_{\delta}$. It is given by

$$
t_{K \mathrm{c}}=\left[\left(1-\frac{1}{e^{2}}\right) \frac{K^{2} D_{0}^{2}}{8 \hbar^{2} \gamma}\right]^{-1} .
$$

Then, for $t_{\delta}>t_{K \mathrm{c}}$, the contribution of $t_{K}$ to the time $t_{\mathrm{r}}$ is greater than the tunneling time $t_{\delta}$. It is also easy to see that for $D_{0}=1$ and $K \gtrsim 1.53 \hbar \gamma$, we obtain $t_{K}>t_{\gamma}$. Table 1 shows, for $D_{0}=1$ (initial chiral purity), the order of magnitude of the values of $t_{\gamma}$ and $t_{K}$, for different values of $t_{\delta}, \gamma$ (with $\gamma \gg t_{\delta}^{-1}$ ) and $K$. The decay rate $\gamma$, related to the frequency of elastic collisions, could be assumed to be of the order of $10^{11}-10^{12} \mathrm{~s}^{-1}$ for a gas at normal conditions, and of the order of $10^{8} \mathrm{~s}^{-1}$ for a gas at very low pressure and ambiance temperature. The value of $K$ can be expected to be comprised in a large range of values, depending, among others, on the density of the gas. ${ }^{23}$ Here we take values of $K$ of the order of $10^{-5}-10^{-6} \mathrm{eV}$. With respect to $\delta$, it is known that there is a large range of possible values. $^{15}$

Only for the values displayed in the first row of the table, $t_{K}$ results lower than $t_{\delta}$, corresponding to a case in which $t_{\delta}<t_{K \mathrm{c}}$. For the rest of the values, the most remarkable result is that, for a large range of values, the times $t_{\gamma}$ and $t_{K}$ are many orders of magnitude greater than $t_{\delta}$, leading to an almost complete 
Table 1 Several values of $t_{\delta} \equiv \hbar / \delta, \gamma$ (with $\gamma \gg t_{\delta}^{-1}$ ) and $K$, from which the orders of magnitude of $t_{\gamma}$ and $t_{K}$ are obtained for $D_{0}=1$. The time $t_{\mathrm{r}}$ for $\varepsilon=0$ is $t_{\mathrm{r}}=t_{\gamma}+t_{K}$

\begin{tabular}{lllll}
\hline$t_{\delta}(\mathrm{s})$ & $\gamma\left(\mathrm{s}^{-1}\right)$ & $K(\mathrm{eV})$ & $t_{\gamma}(\mathrm{s})$ & $t_{K}(\mathrm{~s})$ \\
\hline $10^{-9}$ & $10^{12}$ & $10^{-5}$ & $10^{-7}$ & $10^{-11}$ \\
$10^{-4}$ & $10^{9}$ & $10^{-5}$ & 1 & $10^{2}$ \\
$10^{-3}$ & $10^{8}$ & $10^{-6}$ & 10 & $10^{3}$ \\
$10^{-3}$ & $10^{11}$ & $10^{-5}$ & $10^{4}$ & $10^{2}$ \\
1 & $10^{8}$ & $10^{-6}$ & $10^{7}$ & $10^{9}$ \\
1 & $10^{11}$ & $10^{-5}$ & $10^{10}$ & $10^{8}$ \\
\hline
\end{tabular}

stabilization of the initial chiral state. The huge values $\left(10^{9}, 10^{10} \mathrm{~s}\right)$ of the time $t_{\mathrm{r}}$ in cases with $t_{\delta} \sim 1 \mathrm{~s}$ are noticeable.

The terms dependent on $\varepsilon$ in eqn (10) contribute to $t_{\mathrm{r}}$ in a very similar way as the evaluated term $t_{K}$ does when $D_{0}=1$. If $\varepsilon$ is the intrinsic parity violating energy, of the order of $10^{-15} \mathrm{eV}$, its contribution to $t_{\mathrm{r}}$ is evidently negligible compared with $t_{K}$ and $t_{\gamma}$. However, other chiral influences coming from external fields could be considered.

The solution for the population difference $D(t)$ is easily obtained from eqn (9) (valid for $\gamma \gg t_{\delta}^{-1}$ ) in the case $K=0$, and reads $D(t)=D_{0} e^{-t / t_{\gamma}}$ with $t_{\mathrm{r}}=\frac{\varepsilon^{2}}{\delta^{2} \gamma}+\frac{\gamma t_{\delta}^{2}}{4}$. For $K \neq 0$, the solution $D(t)$ is easily obtained by means of a $t-D$ plot of eqn (9), as is shown in Fig. 1 , for the case $\varepsilon=0, t_{\delta}=10^{-4} \mathrm{~s}$ and $\gamma=10^{9} \mathrm{~s}^{-1}$. In this figure we observe the nonlinear effect of $K$ on the decay of $D(t)$ which is far from an exponential decay and becomes slower than the decay obtained for $K=0$, the racemization time being of the order of $100 \mathrm{~s}$ or more. We recall that the coherence is completely lost when $D=0$. We note that for $\varepsilon=0$ the results are independent of the sign of $K$.

In Fig. 2 we observe the effect of a possible external chiral influence so that $\varepsilon=2 \times 10^{-6} \mathrm{eV}$, with the same values for the other parameters used in Fig. 1. The increase in the time decay (note that the values on the $X$-axis are higher than in Fig. 1) is remarkable. If $K$ and $\varepsilon$ have different sign, the decay is somewhat faster than in the case they have the same sign, as can be inferred from eqn (10). If $\varepsilon$ is greater enough, $D(t)$ tends to exhibit an exponential decay, as expected from inspection of eqn (9).

In all the cases analyzed above, $D(t)$ does not oscillate due to the high enough $\gamma$ compared with $t_{\delta}^{-1}$. When the solutions $D(t)$, shown in Fig. 1 and 2, are compared with the exact solutions obtained numerically from eqn (6), (7), it can be seen that if $\gamma$ is only five times greater than $t_{\delta}^{-1}$, the approximate solutions differ from the exact ones by less than 0.1 per cent.

- Case $\gamma \lesssim \delta / \hbar$. Let us now analyze the case in which $\gamma$ is of the order of or lower than $t_{\delta}^{-1}$, which means that the average time between collisions is longer than or of the order of the tunneling time $t_{\delta}=\hbar / \delta$. In this case, the solution $D(t)$ as well as the coherence $\rho_{\mathrm{LR}}(t)$ are obtained numerically from eqn (6), (7), and shown in Fig. 3 for several cases.

First we consider $\varepsilon=0$ and $\gamma=0$. For $K / \delta \gtrsim 4, \operatorname{Vardi}^{14}$ found that the population is trapped in the initial chiral state, while for lower values of $K / \delta, D(t)$ oscillates around $D=0$. For the initial condition $\rho_{\mathrm{LL}}-\rho_{\mathrm{RR}}=1$, we show in Fig. 3(a) and (b) the case for $K / \delta=10$, which exhibits the same oscillating
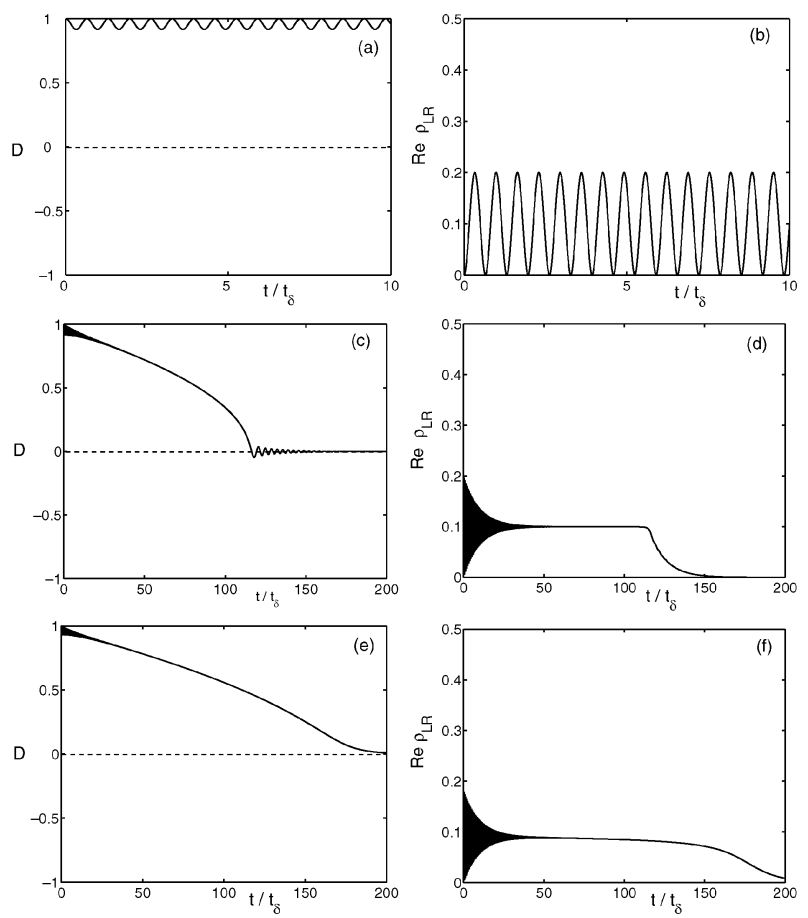

Fig. 3 For $K / \delta=0$, population difference $D \equiv \rho_{\mathrm{LL}}(t)-\rho_{\mathrm{RR}}(t)$ and the real part of the coherence $\rho_{\mathrm{LR}}$ versus the normalized time $t / t_{\delta}$, in cases (a) and (b): $\gamma=0$ and $\varepsilon / \delta=0$; (c) and (d): $\gamma t_{\delta}=0.1$ and $\varepsilon / \delta=0$; (e) and (f): $\gamma t_{\delta}=0.1$ and $\varepsilon / \delta=0.5$. The oscillations of $D$ in (c) and (e), and coherence in (d) and (f), at short times, cannot be resolved at the scale of the figure.

behavior for all times. When the collisional decoherence is introduced, the solutions are of the type obtained for $\gamma t_{\delta}=0.1$, shown in Fig. 3(c) and (d). We see that the population difference, $D$, first decays with small oscillations (scarcely seen in the figure), but without changing the sign of the chirality of the sample. However, for longer times, the regime imposed by $\gamma$ dominates, leading unavoidably to racemization since $D=0$ is reached at the same time in which coherence is lost $\left(\rho_{\mathrm{LR}}=0\right)$. This is obtained in a time longer than tunneling (two orders of magnitude greater than $t_{\delta}$ in this case). Correspondingly, the coherence behaves differently for short times and for long times. For a lower $\gamma t_{\delta}$ value, or (and) a higher $K / \delta$ value, the decay is much more slower. For example, for the same values as before except that $K / \delta=20$, the racemization time results to be three orders of magnitude greater than $t_{\delta}$. This result is also obtained for the same values as in Fig. 3(c) and (d) except that $\gamma t_{\delta}=0.01$. However, the ratio $t_{\mathrm{r}} / t_{\delta}$ is much lower than in the case where $\gamma \gg \delta / \hbar$. Finally, if there is a small chiral influence such that $\varepsilon / \delta=0.5$ for example, the solutions decay slower than in the cases (c) and (d), as shown in Fig. 3(e), (f).

\section{Conclusion}

We have studied the temporal evolution of the molecular state of a chiral molecule in which both chiral enantiomers $L$ and $R$ are only connected by tunneling. We have considered a decoherence effect due to the "dephasing" induced by collisions in tunnel dynamics, together with the effect of 
a mean-field generated by the environment where an energetic difference, $K$, between homochiral and heterochiral interactions is assumed. A possible slight asymmetry in the double well has also been considered. We have shown that the presence of the mentioned decoherence leads unavoidably to equal populations of $|L\rangle$ and $|R\rangle$ chiral states, even for a high enough value of $K$ which tends to keep localized an initial chiral state. This result is in contrast with the case where this decoherence effect is absent. ${ }^{13,14}$

In the case in which the decoherence rate is much greater than the tunneling rate, it is found that the known stabilization of a chiral state promoted by collisional decoherence could be enhanced and even dominated by the contribution of $K$, the stabilization time being many orders of magnitude larger than the tunneling time for a wide range of the parameter values. An estimation of this stabilization time is made. A critical tunneling time above which the stabilization time induced by $K$ is longer than tunneling is obtained.

In the case in which the decoherence decay rate is smaller than or of the order of the tunneling rate, the effect of a high enough value of $K$ to stabilize an initial chiral state ${ }^{14}$ becomes drastically reduced by the collisional decoherence, but in spite of it, a stabilization of the initial chiral state for times longer than tunneling is obtained. However, these times are not so long as in the preceding case.

One of the authors (I. G.) acknowledges fruitful discussions with A. S. Sanz and F. Carreño. This work was supported by MICINN (Spain) through projects FIS2010-22082, FIS201021372, and through the Juan de la Cierva program (P. B.).

\section{References}

1 F. Hund, Z. Phys., 1927, 43, 805.

2 M. Simonius, Phys. Rev. Lett., 1978, 40, 980.

3 R. A. Harris and L. Stodolsky, J. Chem. Phys., 1981, 74, 2145.

4 R. A. Harris and R. Silbey, J. Chem. Phys., 1983, 78, 7330.

5 R. A. Harris and R. Silbey, J. Chem. Phys., 1985, 83, 1069.

6 R. Silbey and R. A. Harris, J. Phys. Chem., 1989, 93, 7062.

7 J. Trost and K. Hornberger, Phys. Rev. Lett., 2009, 103, 023202.

8 P. W. Anderson, Phys. Rev., 1949, 75, 1450.

9 H. Margenau, Phys. Rev., 1949, 76, 1423.

10 P. Claverie and G. Jona-Lasinio, Phys. Rev. A, 1986, 33, 2245.

11 M. Cattani, J. Quant. Spectrosc. Radiat. Transfer, 1991, 46, 507.

12 M. Cattani and J. M. F. Bassalo, J. Quant. Spectrosc. Transfer, 2006, 102, 441.

13 G. Jona-Lasinio, C. Presilla and C. Toninelli, Phys. Rev. Lett., $2002,88,123001$.

14 A. Vardi, J. Chem. Phys., 2000, 112, 8743.

15 M. Quack, J. Stohner and M. Willeke, Annu. Rev. Phys. Chem., 2008, 59, 741 .

16 P. Bargueño, I. Gonzalo and R. Pérez de Tudela, Phys. Rev. A, 2009, 80, 012110

17 P. Bargueño, I. Gonzalo, R. Pérez de Tudela and S. Miret-Artés, Chem. Phys. Lett., 2009, 486, 204.

18 I. Gonzalo, P. Bargueño, R. Pérez de Tudela and S. Miret-Artés, Chem. Phys. Lett., 2010, 489, 127.

19 B. Darquié, C. Stoeffler, A. Shelkovnikov, C. Daussy, et al., Chirality, 2010, 22, 870.

20 P. Bargueño, R. Pérez de Tudela, I. Gonzalo and S. Miret- Artés, Phys. Chem. Chem. Phys., 2011, 13, 806.

21 P. Bargueño, I. Gonzalo and S. Miret-Artés, Phys. Chem. Chem. Phys., 2011, 13, 850.

22 R. Bast, A. Koers, A. Severo Pereira Gomes, M. Ilias, et al., Phys. Chem. Chem. Phys., 2011, 13, 864.

23 M. J. Vlot, J. C. van Miltenburg and H. A. Oonk, J. Chem. Phys., 1997, 107, 101012. 